\title{
AGILE detection of delayed gamma-ray emission from GRB 080514B
}

\author{
A. Giuliani ${ }^{1}$, S. Mereghetti ${ }^{1}$, F. Fornari ${ }^{1}$, E. Del Monte ${ }^{3}$, M. Feroci ${ }^{3}$, M. Marisaldi ${ }^{5}$, P. Esposito ${ }^{1,7,18}$, F. Perotti ${ }^{1}$, \\ M. Tavani ${ }^{3,4}$, A. Argan ${ }^{3}$, G. Barbiellini ${ }^{6}$, F. Boffelli ${ }^{7,18}$, A. Bulgarelli ${ }^{5}$, P. Caraveo $^{1}$, P. W. Cattaneo ${ }^{7}$, A.W. Chen ${ }^{1,2}$, \\ E. Costa ${ }^{3}$, F. D’Ammando ${ }^{3,4}$, G. Di Cocco ${ }^{5}$, I. Donnarumma ${ }^{3}$, Y. Evangelista ${ }^{3}$, M. Fiorini ${ }^{1}$, F. Fuschino ${ }^{5}$, M. Galli ${ }^{14}$, \\ F. Gianotti ${ }^{5}$, C. Labanti ${ }^{5}$, I. Lapshov ${ }^{3}$, F. Lazzarotto ${ }^{3}$, P. Lipari ${ }^{12}$, F. Longo ${ }^{6}$, A. Morselli ${ }^{9}$, L. Pacciani ${ }^{3}$, A. Pellizzoni ${ }^{1}$, \\ G. Piano ${ }^{3,4}$, P. Picozza ${ }^{9}$, M. Prest ${ }^{10}$, G. Pucella ${ }^{3}$, M. Rapisarda ${ }^{11}$, A. Rappoldi ${ }^{7}$, P. Soffitta ${ }^{3}$, M. Trifoglio ${ }^{5}$, A. Trois ${ }^{3}$, \\ E. Vallazza ${ }^{6}$, S. Vercellone ${ }^{1}$, D. Zanello ${ }^{12}$, L. Salotti ${ }^{13}$, S. Cutini $^{8}$, C. Pittori ${ }^{8}$, B. Preger ${ }^{8}$, P. Santolamazza ${ }^{8}$, \\ F. Verrecchia ${ }^{8}$, N. Gehrels ${ }^{15}$, K. Page ${ }^{16}$, D. Burrows ${ }^{17}$, A. Rossi ${ }^{19}$, K. Hurley ${ }^{20}$, I. Mitrofanov ${ }^{21}$, and W. Boynton ${ }^{22}$ \\ (Affiliations can be found after the references)
}

Received 1 August 2008 / Accepted 8 September 2008

\section{ABSTRACT}

GRB 080514B is the first gamma ray burst (GRB), since the time of EGRET, for which individual photons of energy above several tens of MeV have been detected with a pair-conversion tracker telescope. This burst was discovered with the Italian AGILE gamma-ray satellite. The GRB was localized by a cooperation between AGILE and the interplanetary network (IPN). The gamma-ray imager (GRID) estimate of the position, obtained before the SuperAGILE-IPN localization, is found to be consistent with the burst position. The hard X-ray emission observed by SuperAGILE lasted about $7 \mathrm{~s}$, while there is evidence that the emission above $30 \mathrm{MeV}$ extends for a longer duration (at least $13 \mathrm{~s}$ ). Similar behavior has been seen from a few other GRBs observed with EGRET. However, during the brightest phases, the latter measurements were affected by instrumental dead time effects, resulting in only lower limits to the burst intensity. Thanks to the small dead time of the AGILE/GRID we could assess that in the case of GRB 080514B the gamma-ray to X-ray flux ratio changes significantly between the prompt and extended emission phase.

Key words. gamma rays: bursts

\section{Introduction}

Only a relatively small number of gamma-ray bursts (GRBs) have been detected so far at energies above the $\mathrm{MeV}$ region. The largest sample of high-energy observations has been collected using the large calorimeter of the EGRET instrument on board the Compton Gamma Ray Observatory (CGRO). This detector provided GRB light curves and spectra in the $1-200 \mathrm{MeV}$ energy range, thus extending the spectral coverage of the brightest bursts discovered at lower energy with the Burst And Transient Source Experiment (BATSE, Kaneko et al. 2008). Only for a handful of GRBs were high-energy photons also detected with the EGRET spark chamber, sensitive in the $30 \mathrm{MeV}-10 \mathrm{GeV}$ energy range (see, e.g. Dingus 2001, and references therein). These observations showed the existence of a few bursts with very hard spectra, with a peak energy of up to $\gtrsim 170 \mathrm{MeV}$, and possibly of GRBs with high energy "excesses", i.e. an emission above $100 \mathrm{MeV}$ greater than the extrapolation of their $\mathrm{X}$-ray spectra. It is particularly important for theoretical models to understand whether the high-energy emission is a separate spectral component, possibly with a different time evolution to that of the prompt emission. This possibility is supported by the slow time decay of the high-energy flux in GRB 941017 (Gonzalez et al. 2003), as well as by the EGRET detection of delayed photons from GRB 940217 (Hurley et al. 1994), including one with an energy of $18 \mathrm{GeV}$ after $1.3 \mathrm{~h}$. Two factors that affect the study of GRBs at high energy are intrinsic to gamma-ray imagers of the old generation, based on spark chamber trackers: the relatively small field of view, limiting the sample of observed GRBs, and the significant instrumental dead time, reducing the number of detected photons in the brightest parts of the bursts, thus preventing a reliable measure of their peak flux and fluence.

Both limitations are overcome by new gamma-ray satellites using self-triggering trackers based on silicon microstrip technology, like AGILE (Tavani et al. 2008). The AGILE gamma-ray imaging detector (GRID), operating in the $30 \mathrm{MeV}-50 \mathrm{GeV}$ energy range, has a field of view as large as one fifth of the whole sky and a dead time of only $\sim 200 \mu$ s, which makes it particularly suited for the observation of GRBs. For comparison, EGRET had a field of view of $\sim 1 \mathrm{sr}$ and a dead time of $\sim 200 \mathrm{~ms}$. Furthermore, AGILE carries other detectors that allow the study of GRB emission in different energy ranges: SuperAGILE provides two onedimensional images (along orthogonal directions) and light curves and spectra in the 17-50 keV range (Feroci et al 2007). The MiniCalorimeter (MCAL, Labanti et al. 2008), besides being used as part of the GRID, can be used to autonomously detect and study GRBs in the $0.35-100 \mathrm{MeV}$ range. Finally, GRB light curves in the hard X-ray band can be obtained from the GRID anti coincidence scintillator panels (ACS, Perotti et al. 2006). The SuperAGILE capabilities in terms of rapid and accurate localizations of GRBs have been well demonstrated with the discovery of its first GRB, 070724B (Del Monte et al. 2008) and this continues with a rate of about 1 GRB per 1-2 months. Instead, MCAL detects approximately 1 GRB per week, as described in Marisaldi et al. (2008). 


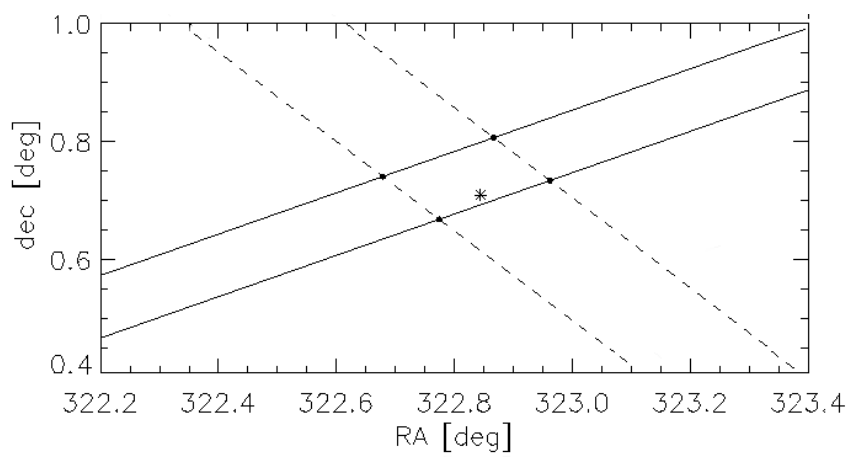

Fig. 1. Combined SuperAGILE and IPN localization of GRB 080514B. The star $(*)$ marks the position of the afterglow found in X-rays by the Swift-XRT (see Page et al. 2008) and in the optical by the IAB80 telescope (see de Ugarte Postigo et al. 2008a,b), the solid lines indicate the SuperAGILE error box and the dashed lines represent the IPN triangulation based on SuperAGILE and Mars Odyssey data.

Here we present the observation of the first GRB for which a positive detection has been obtained by the GRID. Interestingly, the AGILE data give strong evidence that the GRB duration at energies above $30 \mathrm{MeV}$ is at least double that observed at lower energies, suggesting that different emission processes might be at work in the gamma and X-ray range.

\section{Observations and data analysis}

\subsection{SuperAGILE}

SuperAGILE detected GRB 080514B on 2008 May 14 at 09:55:56 UT (hereafter $t_{0}$ ). The GRB triggered the on-board detection system (Del Monte et al. 2007) and was localized within the 1D region of the SuperAGILE field of view. The detection was confirmed by the on-ground trigger, yielding an average signal-to-noise ratio of 15.2 on a $4.096 \mathrm{~s}$ timescale. The burst was at an off-axis angle of $-37.61^{\circ}$ along the $X$ coding direction, and outside the field of view in the $Z$ direction. This resulted in an error box consisting of a long and narrow (15 arcmin total width) rectangle centered at RA $=322.104^{\circ}$, Dec. $=0.486^{\circ}(\mathrm{J} 2000)$, and extending toward $\mathrm{RA}=302.9^{\circ}$, Dec. $=-6.2^{\circ}$ and $\mathrm{RA}=341.3^{\circ}$, Dec. $=+7.1^{\circ}$. GRB 080514B was also detected by Mars Odyssey, MESSENGER, Konus-Wind, Suzaku/wideband allsky monitor (WAM), and INTEGRAL/SPI-anticoincidence shield in the Interplanetary Network for GRB localization. Initially, an Odyssey-SuperAgile annulus was obtained (Rapisarda et al. 2008). Combining the SuperAGILE error box with the IPN annulus gives the error region, with an area of about 100 square arcmin, shown in Fig. 1.

Two observations of the afterglow of GRB 080514B with the Swift X-Ray Telescope (XRT; Burrows et al. 2005) were obtained as a target of opportunity observation. A bright fading source was detected inside the SuperAGILEIPN error box (Page et al. 2008) An optical afterglow was discovered by observing the complete IPN error box (de Ugarte Postigo et al. 2008a), and the probable host galaxy was identified by Perley et al. (GCN 7874), who constrained the redshift to be $<2.3$.

The SuperAGILE lightcurve of GRB 080514B in the 17-50 keV energy band is shown in the bottom panel of Fig. 2. The burst has a duration of $\sim 7 \mathrm{~s}$ and a multipeak structure.

From the SuperAGILE imaging analysis, under the assumption of a Crab-like spectrum, we can derive an average flux of

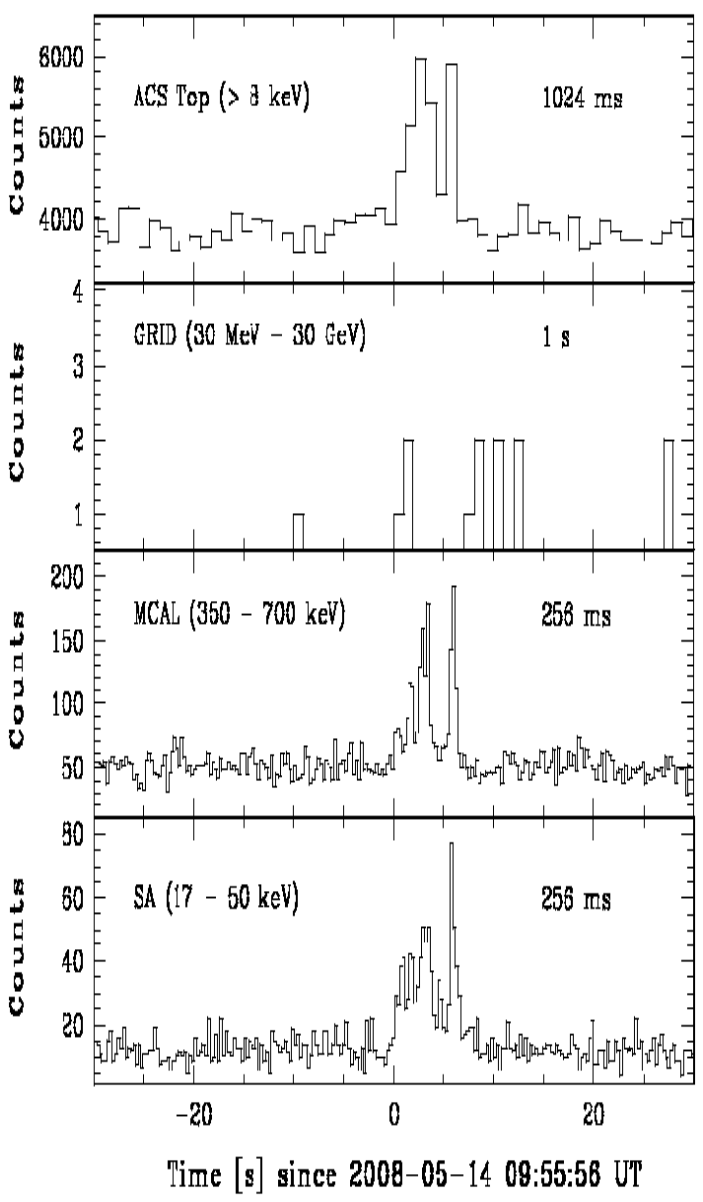

Fig. 2. Lightcurves of GRB 080514B as measured with the different detectors on board the AGILE satellite. The SuperAGILE light curve was obtained selecting only the illuminated region of the detector.

$2.3 \times 10^{-7} \mathrm{erg} \mathrm{cm}^{-2} \mathrm{~s}^{-1}$, a fluence of $1.6 \times 10^{-6} \mathrm{erg} \mathrm{cm}^{-2}$, and a $256 \mathrm{~ms}$ peak flux of $7.3 \times 10^{-7} \mathrm{erg} \mathrm{cm}^{-2} \mathrm{~s}^{-1}$ (all the values refer to the $17-50 \mathrm{keV}$ energy band). The spectra, for the time interval from $t_{0}$ to $t_{0}+7 \mathrm{~s}$, were accumulated separately in the two illuminated detectors and fitted simultaneously. A power law adequately describes the data in the $20-50 \mathrm{keV}$ energy band, with a photon index of $-0.02_{-0.48}^{+0.47}$ (at a $90 \%$ confidence level, with $\chi_{r}^{2}=0.85$ for 23 degrees of freedom). Based on the best fit model, the average flux and the fluence are in reasonable agreement with the values extracted from the images and are in good agreement with the extrapolation of the Konus-Wind values (Golenetskii et al. 2008).

\subsection{MCAL detection}

GRB 080514B also triggered the AGILE/MCAL detector. A valid trigger was issued by the onboard trigger logic at $t_{0}$, after a simultaneous partial trigger was detected, in the $1.024 \mathrm{~s}$ time window, by two of the nine detector ratemeters (see Fuschino et al. 2008, for a detailed description of the MCAL burst trigger logic). MCAL data were collected in photon-byphoton mode for the time interval between $100 \mathrm{~s}$ before and $40 \mathrm{~s}$ after the trigger time, yielding energy information and a timetag with $2 \mu$ s accuracy for each event.

The MCAL lightcurve (Fig. 2) shows a multi-peaked structure and a total duration (T90) of about $5.6 \mathrm{~s}$. According to preliminary in-flight calibration parameters, the time-integrated spectrum between $t_{0}$ and $t_{0}+7 \mathrm{~s}$ can be fitted with a power law 
with photon index $2.6 \pm 0.2$. The fluence in the $500-5000 \mathrm{keV}$ energy range is $(7.8 \pm 1.5) \times 10^{-6} \mathrm{erg} \mathrm{cm}^{-2}$, and the $256 \mathrm{~ms}$ peak flux, measured at $t_{0}+6 \mathrm{~s}$, is $(3.6 \pm 1.1) \times 10^{-6} \mathrm{erg} \mathrm{cm}^{-2} \mathrm{~s}$ in the same energy band. No significant spectral evolution at energies higher than $350 \mathrm{keV}$ is observed. We note that the MCAL photon index is in good agreement with the high energy photon index obtained with Konus-Wind (Golenetskii et al. 2008) and Suzaku/WAM (Hanabata et al. 2008). The observed fluence is also in good agreement with the $S u z a k u / W A M$ results, while it is about $60 \%$ that obtained by Konus-Wind. The MCAL GRB detection capabilities and a discussion on the preliminary in-flight calibration is reported in Marisaldi et al. (2008).

\subsection{GRID: high energy emission}

The AGILE/GRID provides directional, timing and energy information for all the detected events. A series of cuts, applied first on-board (both through hardware-coded and software algorithms) and subsequently in the ground analysis, allows a discrimination between celestial gamma-ray photons and particleinduced or Earth albedo background. In this analysis we used the version F4 of the Standard Analysis filter which first rejects events that can be confidently attributed to background particles and then estimates, through a Kalman filter technique, the energies and arrival directions of the events classified as photons (Giuliani et al. 2006). To search for gamma-ray emission associated with GRB 080514B we used all the events classified as photons with energy $>25 \mathrm{MeV}$. Taking into account the off-axis position and assuming a power-law spectrum with photon index 2, the effective area corresponding to this event selection is about $400 \mathrm{~cm}^{2}$.

Our first search, which led to the preliminary report of a possible detection (Giuliani et al. 2008), was based on the photons collected in 4 time intervals of different durations $(5,10$, 20 and $30 \mathrm{~s}$ ) starting at $t_{0}$ within a radius of 15 degrees centered in the middle of the error region provided by SuperAGILE $\left(\mathrm{RA}=322.15^{\circ}\right.$, Dec. $\left.=0.5^{\circ}\right)$. This extraction radius corresponds to the $68 \%$ containment region for the above energy selection and a "canonical" power law spectrum with photon index $\Gamma=2$. To estimate the number of expected background photons, we used a large annular region and time intervals before and after the GRB in the same AGILE orbit. This yielded an expected rate of 0.233 counts $\mathrm{s}^{-1}$ in the GRB extraction radius ${ }^{1}$. Thus the 8 photons observed in the $10 \mathrm{~s}$ long time interval correspond to a chance probability of about $2.8 \times 10^{-3}$ of being due to the background. The number of photons observed in the 5, 20 and $30 \mathrm{~s}$ long intervals are respectively 4, 16 and 20, and correspond to chance probabilities of $0.031,3.1 \times 10^{-5}$ and $4.4 \times 10^{-5}$.

Independent evidence supporting the gamma-ray detection of GRB 080514B came from the quick look imaging analysis performed immediately after the SuperAGILE localization. This is illustrated in the sky image shown in Fig. 3. The black dots indicate the positions of the photons detected during the whole orbit, while the stars are only those in the $20 \mathrm{~s}$ time interval starting at $t_{0}$. The clustering of the photons (about $2 \times 10^{-2} \mathrm{ph} \mathrm{deg}^{-2}$ in the first $20 \mathrm{~s}$ ) near the center of the long SuperAGILE strip strongly suggested that the burst was indeed located, as later confirmed by the IPN localization.

In order to perform a more refined analysis to derive the GRB spectrum and light curve, we adopt here an energy-dependent

\footnotetext{
1 This value of the expected background rate has been confirmed by the subsequent analysis of longer time spans of data from other AGILE orbits and with the same sky pointing direction.
}

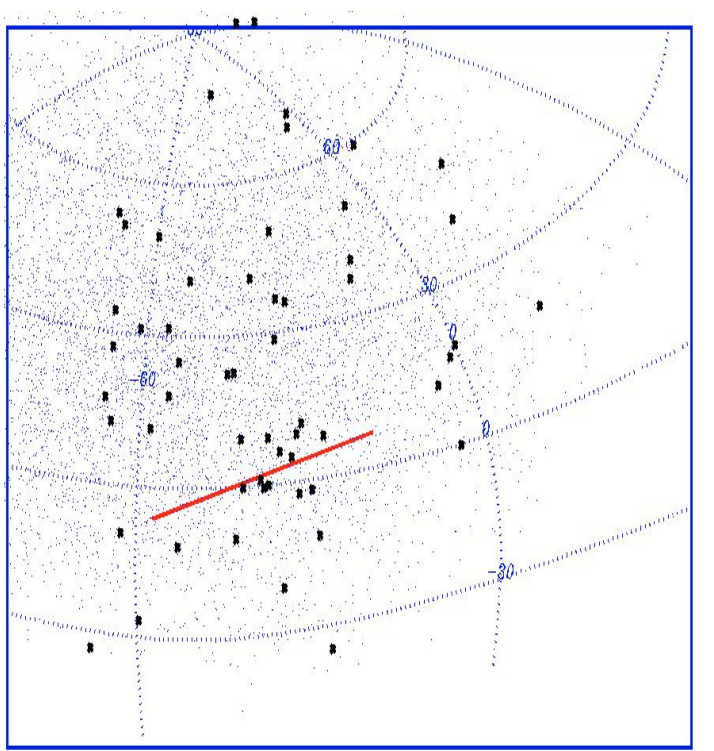

Fig. 3. Distribution in Celestial coordinates of all the GRID photons detected during the orbit in which GRB 080514B was detected ( $23000 \mathrm{~s}$ exposure time). The photons detected from $t_{0}$ to $t_{0}+20 \mathrm{~s}$ are marked by stars. The red line indicates the SuperAGILE error box. The absence of star marks to the left of $l \sim-70$ is due to the Earth occultation during the GRB.

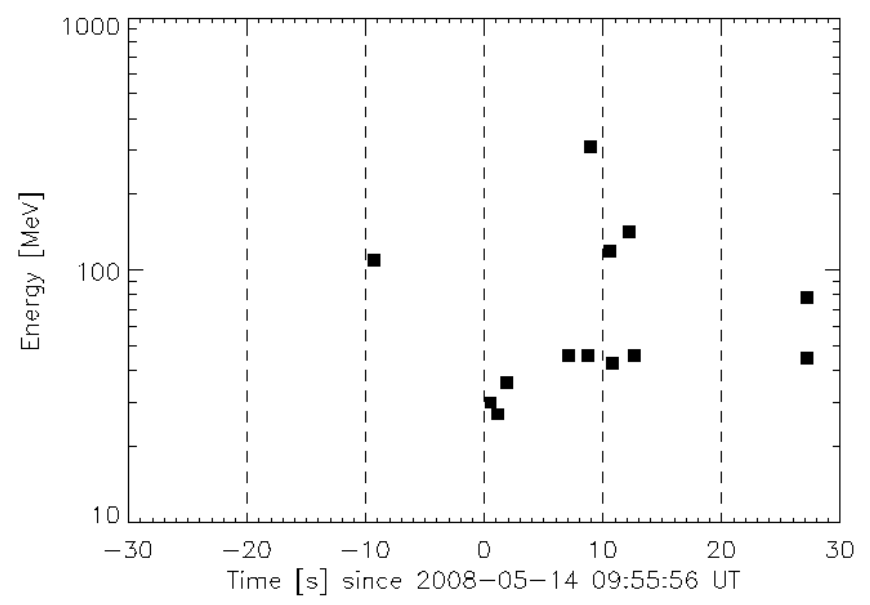

Fig. 4. Energy of the photons detected by the GRID from the direction of GRB 080514B .

extraction radius based on the instrument point spread function ${ }^{2}$. This leads to the selection of events most likely to be associated with the GRB. Their energies and times of arrival are plotted in Fig. 4 while the resulting GRID light curve is shown in Fig. 2. A comparison with the data obtained at lower energies clearly shows that the emission of high energy photons lasted significantly longer. To compare the high-energy light curve, as inferred from the sparse GRID photons, with that observed at lower energy, we estimated whether the time of arrivals of the 10 GRID photons are consistent with the distribution expected from the shape of the MCAL and SuperAGILE light curves. Using a Kolmogorov-Smirnov test, we obtained a probability for this hypothesis of only about 0.003 , confirming that the GRB lasts longer in the GRID energy range. The time of arrivals of the GRID events are instead statistically consistent with a constant

2 The background rate corresponding to this data selection is 0.147 counts $\mathrm{s}^{-1}$, so the statistical significance of the GRID detection is similar to that derived from the quick look analysis reported above. 
flux over the whole time interval from $t_{0}$ to $t_{0}+20 \mathrm{~s}$, contrary to what is seen in all the data at lower energy. In this respect it is important to note that the number of GRID photons in the first time interval, where a brighter emission could be expected, is not limited by dead time effects.

The distribution of the energies of the GRID photons is consistent with a power law spectrum of photon index between 3.5 and 2, in agreement with the value obtained extrapolating the Konus-Wind spectrum (2.5). Assuming a photon index of 2.5, the $25 \mathrm{MeV}-30 \mathrm{GeV}$ fluence in the time interval from $t_{0}$ to $t_{0}+7 \mathrm{~s}$ is $(1.4 \pm 0.7) \times 10^{-2} \mathrm{ph} \mathrm{cm}^{-2}$, while the fluence from $t_{0}$ to $t_{0}+20 \mathrm{~s}$ is $(3.3 \pm 1.1) \times 10^{-2} \mathrm{ph} \mathrm{cm}^{-2}$. Due to the large statistical errors affecting these values, both are consistent with the extrapolation of the spectrum measured at lower energies.

\section{Discussion}

We have reported evidence for high-energy (>25 MeV) emission from GRB 080514B a burst belonging to the class of long duration GRBs, discovered thanks to the complement of detectors on board the AGILE satellite. The highest-energy photon consistent with the direction of the burst has an energy of about $300 \mathrm{MeV}$. Most of the detected GRB photons are at lower energy, in the range $\sim 25-50 \mathrm{MeV}$, consistent with a power law spectrum with a photon index of 2.5. Previous detections of GRBs at these high energies were obtained more than 10 years ago with EGRET, before the discovery of GRB afterglows confirmed their cosmological origin. X-ray and optical/NIR observations indicate that the afterglow properties of GRB 080514B are similar to those of other bursts (Rossi et al. 2008).

The most striking feature of GRB 080514B concerns the fact that the arrival times of the high energy photons detected with the AGILE/GRID do not coincide with the brightest peaks seen in hard X-rays. Three photons are concentrated within $2 \mathrm{~s}$ at the beginning of the burst, while the next ones arrive only when the X-ray emission has returned to a level consistent with the background ( $7 \mathrm{~s}$ after the beginning of the burst). The number of photons in the initial $2 \mathrm{~s}$ is not limited by instrumental dead time effects, as was the case for some of the EGRET GRBs for which only a flux lower limit could be measured, corresponding to the brightest peaks. This implies a rapid time evolution of the gamma-ray to X-ray flux ratio, although a quantitative assessment of this variability is hampered by the small statistics.

Previous EGRET observations indicated a similar behavior for the bursts GRB 941017 (Gonzalez et al. 2003) and GRB 940217 (Hurley et al. 1994), suggesting the possibility that the high-energy emission, at least in some cases, is not a simple extension of the main component, but originates from a different emission mechanism and/or region. Theoretical models predict that Inverse Compton (IC) effect plays a significant role in the high-energy emission from GRBs. The ratio of IC $(\sim \mathrm{MeV}-\mathrm{GeV})$ to synchrotron $(\sim \mathrm{keV})$ fluences depends on the energy of the relativistic electrons accelerated in the shocks and on the ratio between magnetic field and photon densities. Detailed time resolved spectroscopy covering the whole energy range, as would now be possible with further AGILE and GLAST observations (complemented by low energy data from one of the $\mathrm{X}$ and soft gamma-ray experiments currently in orbit), is required to disentangle the different components and obtain some constraints on the physical parameters of the sources.

Acknowledgements. The AGILE Mission is funded by the Italian Space Agency (ASI) with scientific and programmatic participation by the Italian Institute of Astrophysics (INAF) and the Italian Institute of Nuclear Physics (INFN).
K.L.P. acknowledges financial support from STFC. K.H. is grateful for support under the Mars Odyssey Participating Scientist program, JPL grant 1282043.

\section{References}

Burrows, D. N., Hill, J. E., Nousek, J. A., et al. 2005, Space Sci. Rev., 120, 165 Del Monte, E., Costa, E., Di Persio, G., et al. 2007, Frascati Phys. Ser., 45, 201 Del Monte, E., Feroci, M., Pacciani, L., et al. 2008, A\&A, 478, L5 de Ugarte Postigo, A., Castro-Tirado, A., Gorosabel, J., et al. 2008a, GCN, 7719

de Ugarte Postigo, A., Castro-Tirado, A., Gorosabel, J., et al. 2008b, GCN, 7720

Dingus, B. 2001, AIP Conf. Ser., 558, 383

Feroci, M., Costa, E., Soffitta, P., et al. 2007, Nucl. Inst. Meth. A, 581, 728

Fuschino, F., Labanti, C., Galli, M., et al. 2008, Nucl. Inst. Meth. A, 588, 17

Giuliani, A., Cocco, V., Mereghetti, S., et al., 2006, Nucl. Inst. Meth. A, 568, 692

Giulianin, A., Fornari, F., Mereghetti, S., et al. 2008, GCN, 7716

Golenetskii, S., Aptekar, R., Mazets, E., et al., 2008, GCN, 7751

Gonzalez, M. M., Dingus, B. L., Kaneko, Y., et al., 2003, Nature, 424, 749

Hanabata, Y., Uehara, T., Fukazawa, Y., et al. 2008, GCN, 7752

Hurley, K., Dingus, B. L., Mukherjee, R., et al. 1994, Nature, 372, 652

Kaneko, K., González, M. M., Preece, R. D., Dingus, B. L., \& Briggs, M. S. 2008, ApJ, 667, 1168

Kouveliotou, C., Preece, R., Bhat, N., et al. 1994, ApJ, 422, L59

Labanti, C., Marisaldi, M., Fuschino, F., et al. 2006, Proc. SPIE 6266, 62663Q

Labanti, C., Marisaldi, M., Fuschino, F., et al. 2008, Nucl. Inst. Meth. A, submitted

Marisaldi, M., Labanti, C., Fuschino, F., et al. 2008, A\&A, 490, 1151 Page, K. L., Beardmore, A. P., Mereghetti, S., et al. 2008, GCN, 7723 Perley, D. A., Bloom, J. S., Chen, H.-W., et al. 2008, GCN, 7874

Perotti, F., Fiorini, M., Incorvaia, S., et al. 2006, Nucl. Instr. Meth. A, 556, 228 Rossi, A., de Ugarte Postigo, A., Ferrero, P., et al. 2008, A\&A, 491, L29

Rapisarda, M., Costa, E., Del, E. Monte, et al. 2008, GCN, 7715

Tavani, M., Argan, A., Barbiellini, G., et al. 2008, A\&A, submitted [arXiv: 0807.4254]

1 INAF/IASF - Milano, via E. Bassini 15, 20133 Milano, Italy e-mail: giuliani@iasf-milano . inaf.it

2 CIFS-Torino, Viale Settimio Severo 63, 10133 Torino, Italy

3 INAF/IASF - Roma, via del Fosso del Cavaliere 100, 00133 Roma, Italy

4 Dip. di Fisica, Univ. "Tor Vergata”, via della Ricerca Scientifica 1, 00133 Roma, Italy

5 INAF/IASF - Bologna, via Gobetti 101, 40129 Bologna, Italy

6 Dip. di Fisica and INFN Trieste, via Valerio 2, 34127 Trieste, Italy

7 INFN - Pavia, via Bassi 6, 27100 Pavia, Italy

8 ASI - ASDC, via G. Galilei, 00044 Frascati (Roma), Italy

9 INFN - Roma "Tor Vergata", via della Ricerca Scientifica 1, 00133 Roma, Italy

${ }^{10}$ Dip. di Fisica, Univ. dell'Insubria, via Valleggio 11, 22100 Como, Italy

11 ENEA - Roma, via E. Fermi 45, 00044 Frascati (Roma), Italy

12 INFN - Roma "La Sapienza", Piazzale A. Moro 2, 00185 Roma, Italy

13 ASI, Viale Liegi 26, 00198 Roma, Italy

14 ENEA, via Martiri di Monte Sole 4, 40129 Bologna, Italy

15 NASA Goddard Space Flight Center, Greenbelt, MD 20771, USA

16 Department of Physics and Astronomy, University of Leicester, Leicester LE1 7RH, UK

17 Department of Astronomy and Astrophysics, Pennsylvania State University, University Park, PA 16802, USA

18 Dip. di Fisica Nucleare e Teorica, Univ. degli Studi di Pavia, via A. Bassi 6, 27100, Pavia, Italy

19 Thüringer Landessternwarte Tautenburg, Sternwarte 5, 07778 Tautenburg, Germany

${ }^{20}$ University of California, Berkeley, Space Sciences Lab, 7 Gauss Way, Berkeley, CA 94720, USA

21 Institute for Space Research, Profsojuznaja 84/32, Moscow 117997, Russia

22 Lunar and Planetary Laboratory, University of Arizona, Tucson, AZ 85721, USA 